\title{
SMALL SOLUTIONS OF CUBIC CONGRUENCES
}

\author{
TODD COCHRANE
}

(Communicated by William W. Adams)

\begin{abstract}
Let $C(\mathbf{x})$ be a cubic form in $n$ variables over $\mathbf{Z}$ and $p$ be a prime. Then for $0<\sigma<\frac{2}{3}$ the congruence $C(\mathbf{x}) \equiv 0(\bmod p)$ has a nonzero solution $x$ with $\max \left|x_{i}\right| \ll p^{1 / 3+\sigma}$, provided that $n>8 / \sigma$, (where the constant in the $\ll$ depends on $n$ and $\sigma$ ).
\end{abstract}

Let $p$ be a prime and $F_{1}(\mathbf{x}), \ldots, F_{r}(\mathbf{x})$ be forms of odd degrees $\leq d$ over $\mathbf{Z}$ where $\mathbf{x}=\left(x_{1}, x_{2}, \ldots, x_{n}\right)$. Let $|\mathbf{x}|=\max _{1 \leq i \leq n}\left|x_{i}\right|$ and let $\mathbf{F}_{p}$ denote the finite field in $p$ elements. In [4, Theorem 3] Schmidt proves that the system of congruences

$$
F_{1}(\mathbf{x}) \equiv F_{2}(\mathbf{x}) \equiv \cdots \equiv F_{r}(\mathbf{x}) \equiv 0(\bmod p)
$$

has a nonzero solution $\mathbf{x}$ with $|\mathbf{x}| \ll p^{1 / 3+\sigma}$ provided that $n>c(r, d) / \sigma^{2}$, where $c(r, d)$ is an explicitly given constant depending only on $r$ and $d$. In this paper we employ the ideas of Schmidt to obtain a refinement of this result in the case of a single cubic form.

Theorem. Let $C(\mathbf{x})$ be a cubic form over $\mathbf{Z}$ and $p$ be a prime. Then for $0<\sigma \leq$ $\frac{2}{3}$ the congruence $C(\mathbf{x}) \equiv 0(\bmod p)$ has a nonzero solution $\mathbf{x}$ with $|\mathbf{x}| \ll p^{1 / 3+\sigma}$ provided that $n>8 / \sigma$. (The constant in $\ll$ depends on $n$ and $\sigma$.)

We note that the value of $c(1,3)$ given in [4] is $\frac{8}{3}$, and so our result is an improvement on the size of $n$ for $\sigma<\frac{1}{3}$. Also we wish to point out that the exponent $\frac{1}{3}+\sigma$ is certainly not the best possible value for a small solution of a cubic congruence. Indeed, in [2], Schmidt shows that for any $\varepsilon>0$ there exists an $n_{0}=n_{0}(r, d, \varepsilon)$ such that for $n>n_{0}(1)$ has a nonzero solution $\mathbf{x}$ with $|\mathbf{x}| \ll p^{\varepsilon}$.

In [1], Davenport and Lewis introduced the concept of the $h$-invariant of a cubic form. Specifically, for a cubic form $C(\mathbf{x})$ over $\mathbf{F}_{p}$, the $h$-invariant $h=h_{p}(C)$ (relative to the field $\mathbf{F}_{p}$ ) is the smallest positive integer $h$ such that $C(\mathbf{x})$ can be written in the form

$$
C(\mathbf{x})=L_{1}(\mathbf{x}) Q_{1}(\mathbf{x})+L_{2}(\mathbf{x}) Q_{2}(\mathbf{x})+\cdots+L_{h}(\mathbf{x}) Q_{h}(\mathbf{x})
$$

Received by the editors June 30, 1988, and, in revised form, September 25, 1988.

1980 Mathematics Subject Classification (1985 Revision). Primary 11D79; Secondary 11L40. 
where $L_{1}, \ldots, L_{h}$ are linear forms and $Q_{1}, \ldots, Q_{h}$ are quadratic forms over $\mathbf{F}_{p}$. Thus $n-h_{p}(C)$ is the largest dimension of any subspace of $\mathbf{F}_{p}^{n}$ on which $C(\mathbf{x})$ is identically zero. Davenport and Lewis used the $h$-invariant to bound complete exponential sums. Schmidt [3] later extended the definition of an $h$ invariant to an arbitrary system of forms and was able to bound both complete and incomplete exponential sums in terms of $h$. We need the following Lemma, which is a special case of Theorem 3 of [3].

Lemma. The congruence $C(\mathbf{x}) \equiv 0(\bmod p)$ has a nonzero solution $\mathbf{x}$ with $|\mathbf{x}| \ll p^{1 / 3+\sigma}$ provided that $h_{p}(C)>8 / 3 \sigma$.

Proof. Let $h=h_{p}(C)$. Suppose first that $h \leq n / 3+n \sigma$. Then $C(\mathbf{x})$ vanishes on an $n-h$ dimensional subspace of $\mathbf{F}_{p}^{n}$ and this subspace corresponds with a lattice of points in $\mathbf{Z}^{n}$ of volume $p^{h}$ on which $C(\mathbf{x}) \equiv 0(\bmod p)$. Thus, by Minkowski's fundamental theorem from the geometry of numbers, there exists a nonzero point $\mathbf{x}$ with $|\mathbf{x}| \leq p^{h / n} \leq p^{1 / 3+\sigma}$ and $C(\mathbf{x}) \equiv 0(\bmod p)$.

If $h>n / 3+n \sigma$ then the theorem follows from the Lemma and our assumption that $n>8 / \sigma$.

\section{REFERENCES}

1. H. Davenport and D. J. Lewis, Exponential sums in many variables, Amer. J. Math. 84 (1962), 649-665.

2. W. M. Schmidt, Diophantine inequalities for forms of odd degree, Advances in Math. 38 (1980), 128-151.

3. __ Bounds for exponential sums, Acta Arithmetica 44 (1984), 281-297.

4. Small solutions of congruences with prime modulus, Diophantine analysis, Proc. Number Theory Sect. Aust. Math. Soc. Conv. 1985, London Math. Soc. Lect. Note Ser. 109 (1986), $37-66$.

Mathematics Department, Kansas State University, Manhattan, Kansas 66506 